\title{
CS05-03
}

\section{CORE SYMPOSIUM: SUICIDE ACROSS EUROPE. A GP-BASED SUICIDE PREVENTION PROGRAM IN HUNGARY}

\author{
Z. Rihmer ${ }^{1}$, K. Szántó ${ }^{2}$ S. Kalmár ${ }^{3}$, H. Hendin ${ }^{4}$, J. Mann ${ }^{5}$
}

${ }^{1}$ National Institute for Psychiatry and Neurology, Semmelweis Medical University, Budapest, Hungary, ${ }^{2}$ Western Psychiatric Institute and Clinic, Pittsburgh, USA, ${ }^{3}$ Semmelweis Hospital, Kiskunhalas, Hungary, ${ }^{4}$ Suicide Prevention International, ${ }^{5}$ Columbia University, New York, USA

Suicide, particularly in the case of current major depression, is quite common among patients who contact their GPs some weeks or months before their death. However, prior studies have shown that GP's education, regarding the diagnosis and treatment of depressive disorders, can reduce suicide mortality in the given area served by trained GPs. The aim of our present study was to evaluate the effectiveness of a depression-management educational program for GPs in a region with a very high suicide rate (over 50 per 100.000) in Hungary. Twenty-eight GPs and their lead nurses, servicing 73,000 inhabitants in the region of Kiskunhalas, participated the 5-year educational program together with estabilishment of a Depression Outpatient Clinic and psychiatrist telephone consultation service. The annual suicide rate in the Kiskunhalas region decreased from 59.7/100.000 (5-year preintervention average) to $49.9 / 100.000$. This decrease was significantly greater than both the county and whole Hungary ( $p=0.001$ and $p=0.001$, respectively). However, the increase of antidepressant prescription was greater in the intervention region compared with both the county and whole Hungary and in women compared with men $(p=0.02)$. There was no change in alcohol-related deaths or rate of unemployment in the intervention region during the whole study period (1996-2000 vs 2001 and 2005$)$. The findings support earlier studies showing that continuous GP education on diagnosis and treatment of depression is an effective method of suicide prevention. The high importance of alcoholism in local suicides was unanticipated and not addressed, suggesting that optimal suicide prevention plans must also consider major local risk factors. 